Andrews University

Digital Commons @ Andrews University

Honors Theses

Undergraduate Research

4-5-2019

\title{
The Impact of Male-Exposure in the Neuronal Response of the L3 Auditory Intemeuron to Model Calls
}

Shekinah Dosunmu

Andrews University, shekinah@andrews.edu

Follow this and additional works at: https://digitalcommons.andrews.edu/honors

Part of the Biology Commons

\section{Recommended Citation}

Dosunmu, Shekinah, "The Impact of Male-Exposure in the Neuronal Response of the L3 Auditory Intemeuron to Model Calls" (2019). Honors Theses. 201.

https://dx.doi.org/10.32597/honors/201

https://digitalcommons.andrews.edu/honors/201

This Honors Thesis is brought to you for free and open access by the Undergraduate Research at Digital Commons @ Andrews University. It has been accepted for inclusion in Honors Theses by an authorized administrator of Digital Commons @ Andrews University. For more information, please contact repository@andrews.edu. 


\section{J. N. Andrews Honors Program \\ Andrews University}

\section{HONS 497}

Honors Thesis

The impact of male-exposure in the neuronal response of the L3 auditory interneuron to model calls

\section{Shekinah Dosunmu}

$04 / 5 / 2019$

Advisor: Dr. Benjamin Navia

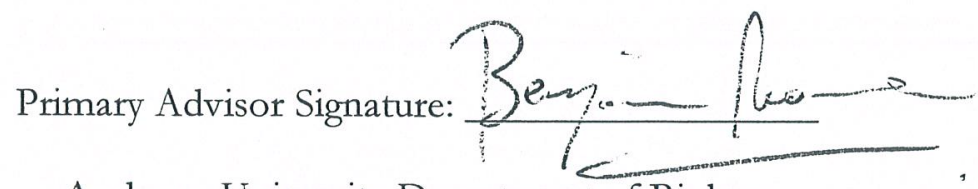

Andrews University Department of Biology 


\begin{abstract}
The L3 auditory neuron in female cricket Acheta domesticus responds to phonotactically attractive calls with decrement: a reduction in the number of action potentials in response to consecutive sound pulses within a chirp. Such unique response has been reported only in virgin females. This study evaluates the response of the L3 in male-exposed females to determine whether they respond to auditory stimuli differently from those exhibited by virgin female crickets.

Preliminary data indicate that L3 in young exposed female crickets responds with different decrement patterns as those reported for virgin females. The implications of these findings are discussèd.
\end{abstract}




\section{Introduction}

Acoustic communication in animals plays a key role in their survival. It serves to mediate mating behavior, foraging behavior, and is used to alert other members of the same species to danger (Siddal \& Marples, 2011). More specifically, auditory signals play a key role in the mating behavior of crickets. In nature, males produce calls to attract female crickets. The movement of the female cricket in response to a male cricket call is referred to as phonotaxis. Positive phonotaxis is the movement of the female cricket towards the conspecfic male call while negative phonotaxis refers to the movement of the female cricket away from the conspecfic male call (Doherty et al, 1985).

The auditory signals produced by the male cricket are detected by the tympanic membranes located on the female cricket's prothoracic legs and processed through the cricket's nervous system. Auditory interneurons within the cricket's prothoracic ganglion in the species $A$. domesticus have been described which play a role in the processing of auditory stimuli. Such neurons include L1, L3, ON1, and ON2 (Atkins et al, 1984). These auditory interneurons receive signals triggered by the male calls and transmit them to the cricket brain through their axonal projections (Atkins et al, 1992). The brain then processes these male calls through its anterior protocerebrum. The neurons within the anterior protocerebrum form an auditory network that is thought to mediate the cricket's phonotactic behavior (Shoneich et al, 2015). Although, the cricket brain does serve as its main processing center for auditory signals, the interneurons located within the prothoracic ganglion also have been shown to play a role in the processing and integration of auditory signals (Atkins et al; 1989).

The response of the auditory interneurons, located within the prothoracic ganglion, to male calls varies due to the different temporal characteristic of the call. The syllable period (SP) is the 
most important temporal parameter of the male's call to elicit the female's phonotactic response (Stout \& McGhee, 1983). The call is composed of three sound pulses with a unique SP; the time between the beginning of one pulse to the beginning of the next. The L3 auditory interneuron shows a more distinct response pattern than any other described auditory interneuron in response to calls that are more phototactically attractive (Atkins et al, 1989).

Specifically, the L3 auditory interneuron exhibits decrement, which has been shown to parallel with phonotaxis and can be used to predict positive phonotaxis in G. bimaculatis. Decrement refers to a decrease in the number of action potentials produced in response to consecutive sound pulses within a chirp. It can be calculated using the following equation: 1- (\# of spikes third pulse / \# of spikes first pulse) *100 (Atkins et al, 1989) and it is represented as a percentage. A higher percent decrement in L3 is associated with positive phonotaxis in virgin female crickets (Samuel et al, 2013). Further studies (Navia et al., 2015), have also indicated that environmental variables like temperature can influence decrement in L3's response to male calls. However, no study has been conducted yet that has explored the potential impact that male exposure can have on the L3's response of female $A$. domesticus.

Samuel et al. (2013), compared the neuronal and phonotactic response of virgin female Gryllus bimaculatus to explore the relationship between percent decrement and positive phonotaxis. This 2013 study helped solidify the understanding of the connection between neuronal and behavioral responses of female crickets. Samuel et al. (2013) found that female crickets showed both positive phonotaxis and a higher percent decrement to SPs of $25-50 \mathrm{~ms}$. The phonotactically attractive range for female G. bimaculatis ranges from $35 \mathrm{~ms}$ to $50 \mathrm{~ms}$ (Samuel et al, 2013). The strong correlation between L3's decrement and positive phonotaxis has not been established for $A$. domesticus crickets in the same way as it has been established for G. bimaculatis. However, previous studies have suggested a parallel between decrement and positive phonotaxis in $A$. domesticus. (Atkins 
et al, 1989). Female crickets of the $A$. domesticus favor the range of $50-70 \mathrm{~ms}$ SP calls (Atkins et al, 1989).

Despite the strong parallel between the phonotactic response and L3's decrement, the temperature study conducted by Navia et al. (2015) has shed light on the impact that environmental conditions can have. This study specifically reported that temperature influences both the phonotactic behavior and the L3's decrement response. In fact, Navia et al. (2015) concludes that temperature affects the percent decrement seen in response to varying male calls of different syllable periods. The study also suggests that temperature fluctuations could be one of the reasons for the variability seen in female's phonotaxis to conspecific male calls. Thus, environmental factors like temperature can have an impact on both the behavioral and neuronal responses.

Additionally, pheromones have been shown to affect phonotactic behavior as well. In a study conducted on crickets of $G$. bimaculatis, it was found that males can distinguish the gender of conspecifics using a channel of communication involving cuticular contact pheromones (Tregenza \& Wedell, 1997). When these contact pheromones were removed, any phonotactic behavior exhibited by the members of these species was abolished (Tregenza \& Wedell, 1997).

All the $A$. domesticus studies mentioned so far have been conducted on virgin female crickets. To better account for possible environmental effects on the processing of calling song recognition such as that of pheromones, this study evaluates the hypothesis that male-exposed female crickets will exhibit a different response of the L3 neuron (measured in decrement) to attractive calls when compared to those seen in previous studies conducted on virgin females. 


\section{Materials and Methodology}

Cricket Care

The handling and maintenance of the crickets is adapted from the procedure as described in Atkins et al (1992). Female $A$. domesticus nymph crickets were obtained from Fluker Farms, Inc. and held in plastic containers. These crickets had access to cricket chow and water. Upon molting, the male and female crickets were placed in containers with appropriate labeling.

Electropbysiological Recordings

Extracellular recordings of the L3 auditory neuron were obtained following the procedure described in Navia et al. (2015). First, female crickets were placed on a wax block. Additional wax was used to secure the crickets to the block ventral side up with the upper thoracic legs positioned on either side of the cricket's head towards the speakers. After which, the cricket was dissected by the careful removal of the thoracic exoskeleton to reveal the prothoracic ganglion and its superior connectives located between the cricket's upper thoracic legs. A saline-filled microsuction electrode was placed on the superior connectives leading from the prothoracic ganglion to the female cricket's brain.

Auditory stimulus

A sequence of computer-generated model calls was played from the speakers located on each side of the cricket using Audacity (version 2.2.1, GPLv2). The calling songs were presented in a non-sequential order $(50 \mathrm{~ms}, 90 \mathrm{~ms}, 70 \mathrm{~ms}, 30 \mathrm{~ms}, 60 \mathrm{~ms}, 80 \mathrm{~ms}, 40 \mathrm{~ms})$ for 30 seconds each with 10 seconds of rest in-between calls.

Characterization of $\mathrm{L} 3$

The L3 neuron was identified with the parameters mentioned in Navia et al. (2015) such as its unique threshold response to sound frequency and intensity and its temporal response to the 
sound stimulus. The L3 neuronal response was then digitized by power lab computer software to generate graphs of the stimulus and the corresponding neuronal response.

Analysis

The digitized neuronal response of the L3 neuron was then used to generate post stimulus time histograms (PST). These PSTs provided the number of action potentials to each sound pulse of the auditory stimulus and allowed the percent decrement of the L3 to each syllable period to be computed. The equation used to compute \% decrement is: 1 - (\# of action potentials for the third sound pulse/ \# of action potentials for the first sound pulse) $* 100$.

To determine if the percent decrement in the response of the L3 neuron in exposed females significantly differed from the one seen in virgin female $A$. domesticus, the percent decrement values for each of the syllable periods tested was averaged. The data for the virgin female were obtained from unpublished data collected in our lab. The average percent decrement values for both, exposed females and virgin females was then plotted in a line graph. Additionally, the mean decrement values for the favorable 50 - 70ms SP range was compared for exposed and virgin female crickets with a standard t-test. The percent decrement for the remaining SPs for exposed and virgin females were compared as well via an independent sample t-test.

\section{Results}

The percent decrement values at different SPs for exposed and virgin $A$. domesticus females were placed into three groups. The first group was that of young exposed females and contained data from four crickets with an age range of three to eight days (this is the data collected in this study). The second group contained data from six young virgin females with ages ranging from five to six days. While the third group contained data from six old virgin females whose age ranged from 20 to 24 days. Data from the second and third groups was collected by Ashley Lynch from our lab 
as part of a previous study (unpublished data). The percent decrement values for different SPs for each of these groups were plotted in a line graph for comparison (Figure 1). In this line graph, there is a lot of overlap in average percent decrement for the 30-50 ms SPs and for the $80-90 \mathrm{~ms}$ SPs (Figure 1). However, the $50-80 \mathrm{~ms}$ SPs for the three groups show differences in average percent decrement values. For young exposed females, the percent decrement peaks in response to $60 \mathrm{~ms}$ SP but decreases by nearly 20 percent for the $70 \mathrm{~ms}$ SP. In contrasts, the old virgin group and the young virgin group show a peak at $70 \mathrm{~ms}$, with the young virgin group displaying a higher percent decrement value than that of the old virgin group (Figure 1).

A t-test was run that compared the percent decrement values in response to the $50-70 \mathrm{~ms}$ SP range between the young exposed females and the old virgin females. Although, the bar graph displayed a slightly higher percent decrement for the old virgin females than that of the young exposed females (Figure 2), no significant difference was found between these two groups $\left(t_{(26)}=\right.$ $-.852, p=.402)$. Additionally, a t-test that compared the percent decrement values in response to the $50-70 \mathrm{~ms}$ SP range between young virgin females and young exposed females was also run (Figure 3). The difference between the percent decrement values between young virgin females and young exposed females was found to be not significant $\left(t_{(26)}=-2.023, p=.053\right)$.

In addition to the t-test run for the most attractive range, further t-tests were run for SP that were out of the $50-70 \mathrm{~ms}$ range (i.e $30,40,80$, and $90 \mathrm{~ms}$ ). Figure 4 displays a bar graph that compares the average percent decrement values in response to SPs that fall out of the 50-70ms range for young exposed females and old virgin females. The bar graph shows a very small difference between the percent decrement values for young exposed and old virgin females (Figure 4). A t-test was run on this group of data and it was found that the percent decrement values in response to SPs that are not in the 50-70ms for young exposed females was not significantly different than that of old virgin females $\left(t_{(37)}=-.707, p=.484\right)$. Similarly, the bar graph comparing the average percent 
decrement values for young exposed and young virgin females in response to SPs outside the 50$70 \mathrm{~ms}$ range shows a very small difference between average decrement values (Figure 5). There is also no significant difference between the percent decrement for young exposed and young virgin females $\left(t_{(36)}=-.793, p=.433\right)$.

\section{Average Decrement Levels Accross Syllable Periods}

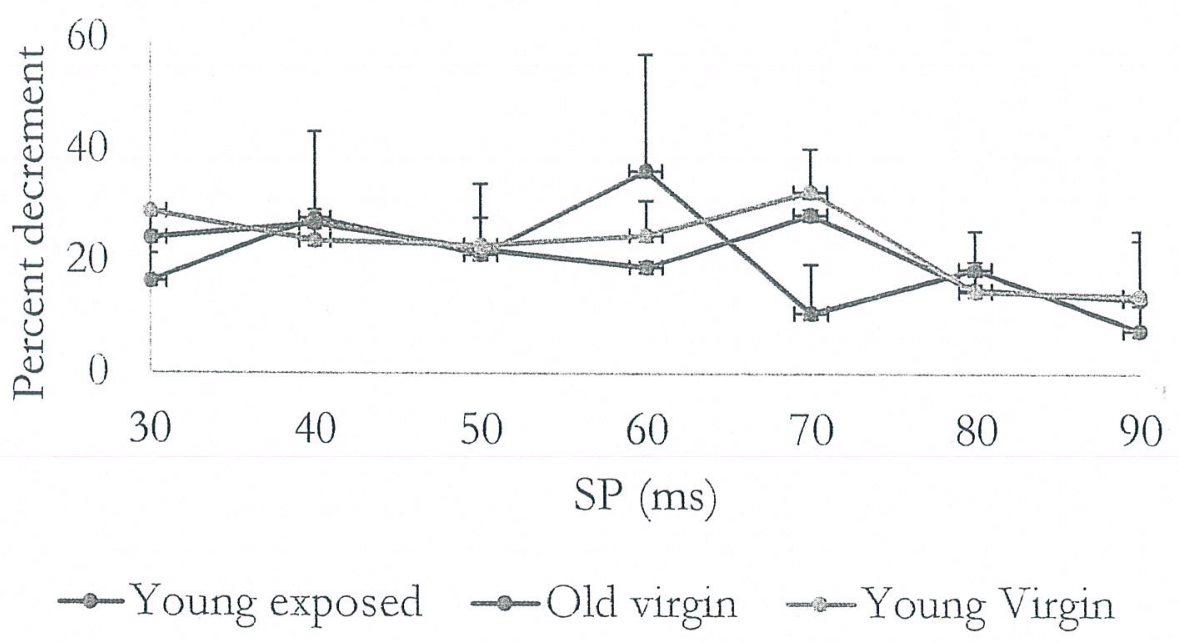

Figure 1. This graph displays the average decrement values for different syllable periods for young exposed female crickets ( $3-8$ day old; $n=4)$, old virgin crickets (20-24 day old; $n=6)$ and young virgin females (5-7 day old; $n=6$ ). The error bars represent the standard deviation for the data sets. 
Average Percent Decrement in the 50-70ms SP

range

40
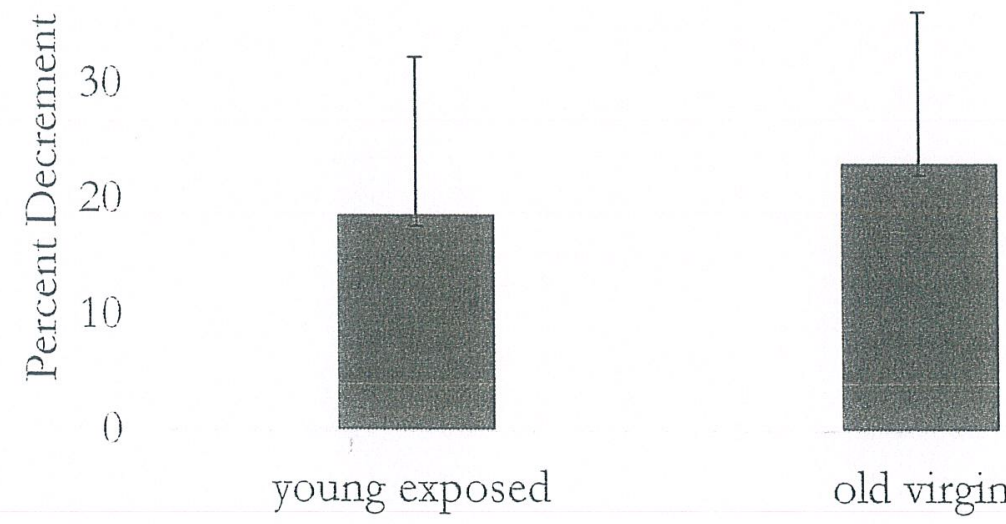

old virgin

Figure 2. These bar graphs display the average percent decrement for young exposed females and old virgin females for the 50-70ms range. The average percent decrement for the young exposed females was $18.72 \%$ while that of the old virgin females was $23.214 \%$. The error bars represent standard deviation. There was no significant difference found between these two data sets $\left(t_{(26)}=\right.$ $-.852, p=.402)$. 


\section{Average Percent Decrement in the 50 ms- 70 ms range}

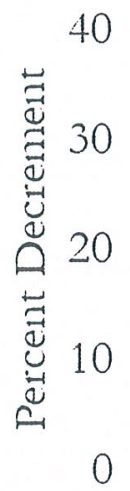

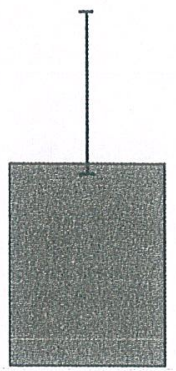

young exposed

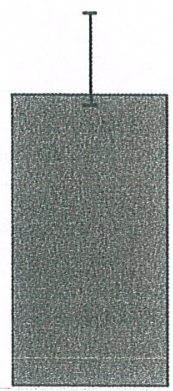

young virgin

Figure 3. These bar graphs display the average percent decrement for young exposed females and young virgin females for the $50-70 \mathrm{~ms}$ range. The average percent decrement for the young exposed females was $18.72 \%$ while that of the old virgin females was $26.7 \%$. The error bars represent standard deviation. There was no significant difference found between these two data sets $\left(t_{(26)}=\right.$ $-2.023, p=.053)$. 
Average Percent decrement for SPs outside the most attractive range

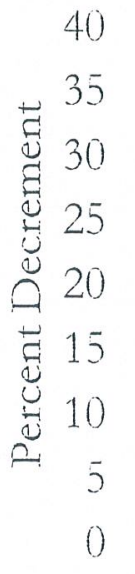

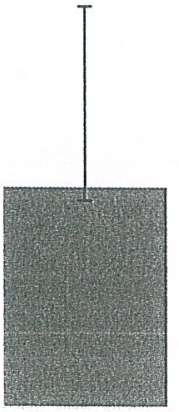

young exposed

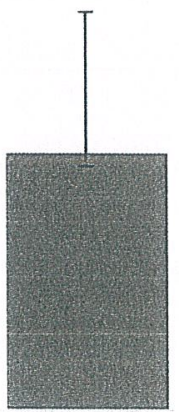

Old virgin

Figure 4. These bar graphs display the average percent decrement for young exposed females and old virgin females for the SPs outside of the 50-70ms range $(30,40,80$, and $90 \mathrm{~ms})$. The average percent decrement for the young exposed females was 17\% while that of the old virgin females was $19.9 \%$. The error bars represent standard deviation. There was no significant difference found between these two data sets $\left(t_{(37)}=-.707, p=.484\right)$. 


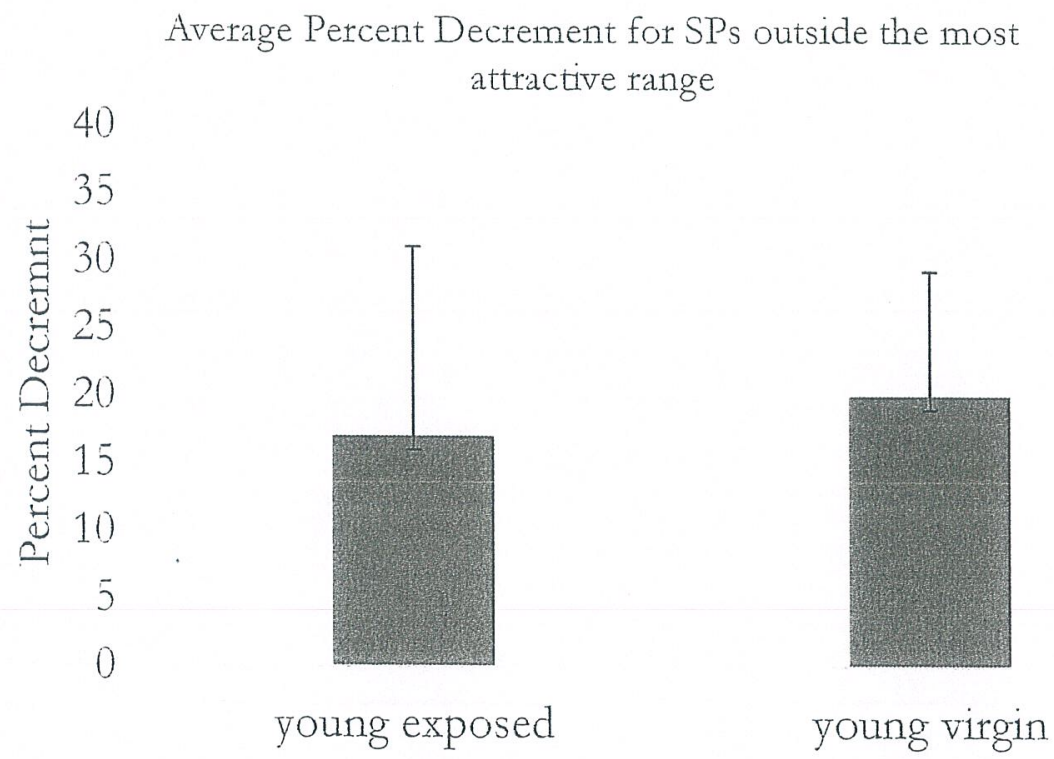

Figure 5. These bar graphs display the average percent decrement for young exposed females and old virgin females for the SPs outside of the 50-70ms range (30,40,80, and 90ms). The average percent decrement for the young exposed females was $17 \%$ while that of the young virgin females was $20 \%$. The error bars represent standard deviation. There was no significant difference found between these two data sets $\left(t_{(36)}=-.793, p=.433\right)$. 


\section{Discussion}

In conclusion, it appears that although there is a difference in the percent decrement values between young exposed females and that of old and young virgin females to SPs within the 50-70ms range, this difference is not significant. Despite this general lack of significant differences however, there was one set of data that came close to being significantly different from each other. When a ttest was run to determine the difference between the percent decrement values in response to the $50-70 \mathrm{~ms}$ range for young exposed females and young virgin females a significance value of $\mathrm{p}=.053$ was obtained. Although, this value is not less than .05 it is quite close. A possible reason for the general lack of significance could be the small sample size of the young exposed females' group. The bar graph of this data also demonstrates a higher percent decrement average for the $50-70 \mathrm{~ms}$ range for young virgin crickets when compared to that of young exposed females (Figure 3). This pattern of decrement is expected as the favorable SP range within female $A$. domesticus is $50-70 \mathrm{~ms}$ (Atkins et $a l, 1989)$.

In addition to the lack of significance differences in the percent decrement values in response to the 50-70 ms range between the three defined groups, there is also no significant difference between the percent decrement values in response to SPs that fall outside of the 50-70ms range for young exposed to young virgin females. Additionally, there is also no significant difference between the percent decrement values in response to SPs outside the attractive range for young exposed and old virgin females. The percent decrement values for all the defined groups are also lower. This is not unexpected however, as the SPs that fall outside of the 50-70 ms range are not as preferred by young virgin females as those that are within the range (Atkins et al, 1989). Thus, it is expected that the percent decrement values would be lower for these ranges.

The difference between the L3 decrement response in young exposed females to that of virgin females illustrated in Figure 1 could be the result of exposure of the young females to 
pheromones. In previous studies, pheromone have been known to play a role in the phonotaxic behavior of crickets (Tregenza \& Wedell, 1997) so it is also possible that the presence of pheromones could be affecting the L3's percent decrement response to model calls. To better isolate the effects of pheromones on the L3's percent decrement response, future studies should control for mating behavior between female and male crickets.

In this study, an independent sample t-test was used to compare the three defined groups for SPs that fell within the attractive range for young virgin female $A$. domesticus of $50-90 \mathrm{~ms}$ and those that did not. Future studies should focus on increasing the sample size of all groups compared in this study. The small $\mathrm{n}$ value in this study prevents any conclusive statements from being made even though the preliminary results seem to support the proposed hypothesis. Another recommendation for future studies would be to assess phonotactic behavior in male exposed females in addition to assessing the L3 electrophysiological response. 


\section{Literature Cited}

Atkins, S., Atkins, G., Rhodes, M. \& Stout, J. (1989). Influence of syllable period on song encoding properties of an ascending auditory interneuron in the cricket Acheta domesticus. Journal of Comparative Physiology A: Neuroetbology, Sensory, Neural, and Bebavioral Physiology, 165, 827 - 836.

Atkins, G., Henley, J., Handysides, R. \& Stout, J. (1992). Evaluation of the behavioral roles of ascending auditory interneurons in calling song phonotaxis by the female cricket (Acheta domesticus). Journal of Comparative Pbysiology A, 170, 363-372.

Atkins, G., Ligman, S., Burghardt, F. \& Stout, J. (1984). Changes in phonotaxis by the female cricket Acheta domesticus L. after killing identified acoustic interneurons. Journal of Comparative Pbysiology A, 154, 795-804.

Doherty, J., \& Hoy, R. (1985). The Auditory Behavior of Crickets: Some Views of Genetic Coupling, Song Recognition, and Predator Detection. The Quarterly Review of Biology, 60(4), $457-472$.

Navia, B., Burden, C., Steely, T., Hasegawa, H., Cha, E., Henson, S. M., Stout, J. \& Atkins, G. (2015). Temperature and its effect on the call and response of the female cricket. Pbysiological Entomology 40, $113-122$.

Samuel, L., Stumpner, A., Atkins, G. \& Stout, J. (2013). Processing of model calling songs by the prothoracic AN2 neurone and phonotaxis are significantly correlated in individual female Gryllus bimaculatus. Physiological Entomology, 38, 344-354.

Shoneich, S., Kostarakos, K., \& Hedwig, B. (2015). An auditory feature detection circuit for sound pattern recognition, Science Advances, 1-7, DOI:10.1126/sciadv.1500325.

Siddal, E., Marples, N. (2011). Hear no evil: The effect of auditory warning signals on avian innate avoidance learned avoidance and memory. Current Zoology, 57, 197-207. 
Stout, J., DeHaan, C. \& McGhee, R. (1983). Attractiveness of the male Acheta domesticus calling song to females. Journal of Comparative Pbysiology A: Neuroetbology, Sensory, Neural, and Bebavioral Pbysiology, 153, 509-521.

Tregenza, A., \& Wedell, N. (1997). Definitive evidence for cuticular pheromones in a cricket. Anim. Behave., 54, 979-984. 